\title{
A Stability Condition with Delay-Dependence for a Class of Switched Large-Scale Time-Delay Systems
}

\author{
Chi-Jo Wang and Juing-Shian Chiou \\ Department of Electrical Engineering, Southern Taiwan University of Science and Technology, No. 1 NanTai Street, \\ Yongkang District, Tainan City, Taiwan \\ Correspondence should be addressed to Juing-Shian Chiou; jschiou@mail.stust.edu.tw
}

Received 4 February 2013; Revised 2 April 2013; Accepted 3 April 2013

Academic Editor: Han H. Choi

Copyright (C) 2013 C.-J. Wang and J.-S. Chiou. This is an open access article distributed under the Creative Commons Attribution License, which permits unrestricted use, distribution, and reproduction in any medium, provided the original work is properly cited.

By using the time-switched method and the comparison theorem, we derived a criterion of delay-dependent stability for the switched large-scale time-delay systems. To guarantee the exponential stability for the switched large-scale time-delay systems with stability margin $\lambda$, the total activation time ratio of the switching law is determined. An example is used to illustrate the effectiveness of our result.

\section{Introduction}

Switched systems can be fully described by a class of continuous-time subsystems (or discrete-time subsystems) and the rule deciding the switching between them. It is most frequent that the switching among multiple subsystems are involved in the control process of practical systems. Many examples with that switching nature can be found in the applications like power systems, chemical process control systems, navigation systems, and automobile speed change systems. In the study of switched systems, most works mainly dealt with stability. In the past twenty years, we have seen increasing interest in the stabilization of such systems; please refer to, for example, [1-4] and the references therein. Two important methods, the state variable methods, and the timeswitched methods are often used to construct the switching law for the switched systems stabilization. The state variable methods look for a common Lyapunov function shared by all subsystems; the overall system stability is assured when such Lyapunov function exits, regardless of the switching strategy selected. However, a common Lyapunov function cannot be easily found; hence, finding of a restricted class of switching laws under which switched systems can be made stable became the focal point. On the other hand, for the time-switched method, the concept of dwell time plays a central role. Recently, there are notable breakthroughs made in several works based on the method of dwell time. It was shown that when all subsystem matrices are Hurwitz, then the overall switched system is always exponentially stable if the time between consecutive switching (dwell time) is sufficiently large. It was demonstrated in [5] that switching among stable linear systems yields a stable system when the switching is "slow-on-the-average." When unstable subsystems of switched systems are present, if the average dwell time is sufficiently large and the total activation time of unstable subsystems is relatively short in comparison, then exponential stability of a desire degree is ensured in [6]. Reference [7] proposed stability conditions using the concepts of minimum/ maximum holding time and redundancy of each subsystem which was involved instead of the multiple Lyapunov functions. A different "time-varying" or "adjustable" dwell time to deal with switched nonlinear systems with disturbances was studied in [8].

It is well known that the presence of time-delays is inevitable in applications like chemical process control, long distance transmission lines, hybrid procedures, and electronic networks. Time delays may lead to poor performances or even instability (see, e.g., $[9,10])$. Hence, we consider the stability issue of switched time-delay systems well worthy of research. Also, a large-scale system is often considered as a class of interconnected subsystems. This aspect reduces the complexity in stability analysis. Recently, different methods 
have been used to investigate the stability and stabilization of large-scale time-delay systems [11]. In view of all these, we consider that the stability of switched large-scale time-delay systems needs to be thoroughly studied [12].

In this paper, we intend to use the time-switched method and the comparison theorem to derive a sufficient stability conditions with delay-dependence for the switched largescale time-delay systems. The total activation time ratio under the switching law is required to be no less than a preset constant. Finally, we will present one simulation example to show the effectiveness of our result. The following notations will be used throughout the paper: $\lambda(A)$ stand for the eigenvalues of matrix $A,\|A\|$ denotes the norm of matrix $A$; that is, $\|A\|=$ $\operatorname{Max}\left[\lambda\left(A^{T} A\right)\right]^{1 / 2} \cdot \mu(A)$ denotes the matrix measure of matrix $A$; that is, $\mu(A)=\operatorname{Max}\left[\lambda\left(\left(A+A^{T}\right) / 2\right)\right]$.

\section{System Description and Problem Statement}

Consider the switched large-scale time-delay systems

$$
\begin{gathered}
\dot{x}_{i}(t)=A_{\delta(t)_{i i}} x_{i}(t)+\sum_{\substack{j \neq i \\
j=1}}^{N} A_{\delta(t)_{i j}} x_{j}(t)+\sum_{j=1}^{N} B_{\delta(t)_{i j}} x_{j}(t-\tau), \\
x_{i}(t)=\psi_{i}(t), \quad t \in[-\tau, 0],
\end{gathered}
$$

where $x_{i}(t) \in R^{n_{i}}$ is the state vectors of the $i$ th subsystem, $i=$ $1,2, \ldots, N, N$ is the number of subsystem for each individual system, $n=\sum_{i=1}^{N} n_{i}$ is the dimension of individual system, $x(t)=\left[\begin{array}{llll}x_{1}^{T}(t) & x_{2}^{T}(t) & \cdots & x_{N}^{T}(t)\end{array}\right]^{T} \in R^{n}$, and $A_{\delta(t) \_i}, A_{\delta(t) \_i j}$ and $B_{\delta(t) i j}$ are matrices of compatible dimensions. $\delta(t)$ : $\left[t_{0}, \infty\right) \rightarrow\{1,2, \ldots, r\}$ is a piecewise constant function of time, called a switch signal, $r$ is the number of individual system; that is, the matrix $A_{\delta(t) \_i}$ switches between matrices $A_{1 i i}, A_{2 i i}, \ldots, A_{r i i}$ belonging to the set $\mathbf{A} \equiv\left\{A_{1_{-} i i}, A_{2 i i}, \ldots, A_{r_{-} i i}\right\}$ and $A_{l_{-} i i}, l \in\{1,2, \ldots, r\}$, the matrix $B_{\delta(t) \_i j}$ switches between matrices $B_{1_{-i j}}, B_{2 \_i j}, \ldots, B_{r_{-} i j}$ belonging to the set $\mathbf{B} \equiv\left\{B_{1_{i j}}, B_{2 i j}, \ldots, B_{r_{-} j}\right\}$ and $B_{l_{-} i j}$, and $\tau>0$ is time-delay duration. $\psi_{i}(t)$ is a vector-valued initial continuous function defined on the interval $[-\tau, 0]$, and finally $\psi_{i}(t)$, defined on $-\tau \leq t \leq 0$, is the initial condition of the state. Assume $r \geq N$.

Hence, the switched large-scale time-delay system (1) can also be written as

$$
\begin{aligned}
\dot{x}_{i}(t)= & A_{l i i} x_{i}(t)+\sum_{\substack{j \neq i \\
j=1}}^{N} A_{l i j} x_{j}(t) \\
& +\sum_{j=1}^{N} B_{l i j} x_{j}(t-\tau) \quad l \in\{1,2, \ldots, r\} .
\end{aligned}
$$

As we have indicated earlier, very few research articles have ever addressed the stability issue of the switched largescale time-delay system (1). Some existing results from the literature are helpful for further stability analysis.

Consider the time-delay system

$$
\dot{x}(t)=A x(t)+B x(t-\tau),
$$

where $x \in R^{n}, A$ and $B$ are matrices in proper dimensions, and $\tau$ is the delay.

Lemma 1 (see [13]). For matrices $A \in R^{n \times n}, B \in R^{n \times n}$, the following relation holds:

$$
\left\|e^{(A+B) t}\right\| \leq e^{\mu(A+B) t}, \quad t \geq 0
$$

Lemma 2 (see [14]). Let $\dot{r}(t) \leq-\alpha r(t)+\beta \sup r(\omega)$ for $t \geq 0$ and if $\alpha>\beta>0$. Then there exist $a \xi>0$ and $a \eta>0$ such that $r(t) \leq \eta e^{-\xi\left(t-t_{0}\right)}$ for $t \geq 0$.

Without loss of generality, we assume that the switched large-scale time-delay system (1) at least has one individual system whose $\mu\left(A_{l_{i i}}+B_{l_{j i}}\right)$ values, $i=1,2, \ldots, N$, are less than zero, and

$$
\begin{aligned}
& \lambda_{i}^{-}=\min _{1 \leq l \leq r_{1}}\left(\left|\mu\left(A_{l_{i i}}+B_{l_{-i i}}\right)\right|\right) \quad \text { when } \mu\left(A_{l_{i i}}+B_{l_{i i i}}\right)<0, \\
& \lambda_{i}^{+}=\max _{r_{1}+1 \leq l \leq r}\left(\mu\left(A_{l_{-i i}}+B_{l i i}\right)\right) \quad \text { when } \mu\left(A_{l_{-i i}}+B_{l i i}\right) \geq 0 .
\end{aligned}
$$

We assumes $T_{i}^{+}(t)$ (or $\left.T_{i}^{-}(t)\right)$ is the total activation time of the $i$ th subsystem whose $\mu\left(A_{l_{-i i}}+B_{l_{i i}}\right)$ values are no less than zero (total activation time of the $i$ th subsystem whose $\mu\left(A_{l_{i i i}}+B_{l_{i i}}\right)$ values are less than zero). The switching law of the $i$ th subsystem can be defined as follows:

$$
\inf _{t \geq t_{0}}\left[\frac{T_{i}^{-}(t)}{T_{i}^{+}(t)}\right] \geq \frac{\lambda_{i}^{+}+\lambda_{i}^{*}}{\lambda_{i}^{-}-\lambda_{i}^{*}}
$$

where $\lambda_{i} \in\left(0, \lambda_{i}^{-}\right), \lambda_{i}^{*} \in\left(\lambda_{i}, \lambda_{i}^{-}\right), 1 \leq i \leq N$.

Moreover, $T^{-}(t)$ (or $T^{+}(t)$ ) is the total activation time of individual systems whose $\mu\left(A_{l_{-i i}}+B_{l_{-} i i}\right)$ values are less than zero for $i=1,2, \ldots, N$ (the total activation time of individual systems at least has one subsystem whose $\mu\left(A_{l i i}+B_{l i i}\right)$ values are not less than zero). The total activation time ratio between $T^{-}(t)$ and $T^{+}(t)$ can be regarded as a switching law of the switched delayed large-scale system (1). Thus, we examine the following problem: finding the total activation time ratio $T^{-}(t) / T^{+}(t)$ which guarantees that the switched large-scale time-delay system (1) is globally exponentially stable with stability margin $\lambda$.

Furthermore, we define an auxiliary matrix as follows:

$$
\dot{y}(t)=C y(t)+\tau E \sup _{t-2 \tau \leq w \leq t} y(w),
$$

where $y(t)=\left[\begin{array}{llll}y_{1}(t) & y_{2}(t) & \cdots & y_{N}(t)\end{array}\right]^{T}, y_{i}(t) \in R, C=$ $\left[c_{i j}\right] \in R^{N \times N}, c_{i i}=-\lambda_{i}, i=j, c_{i j}=a_{i j}^{*}$, and $i \neq j$ for $i, j=$ $1,2, \ldots, N$, where $a_{i j}^{*}=\max _{l=1,2, \ldots, r}\left(\left\|A_{l_{-} i j}+B_{l i j}\right\|\right), E=\left[e_{i j}^{*}\right] \epsilon$ $R^{N \times N}$, and $e_{i j}^{*}=\max _{l=1,2, \ldots, r}\left[\sum_{m=1}^{N}\left(B_{l i m} A_{l \_m j}+B_{l \_i m} B_{l \_m j}\right)\right]$.

A sufficient condition for stability of the switched largescale time-delay systems (1) is established in the following theorem by using the time-switched method. 


\section{Main Results}

Theorem 3. Suppose that the switched large-scale time-delay system (1) exists at least an individual subsystem whose $\mu\left(A_{l_{-i i}}+B_{l_{-i i}}\right)$ value is less than zero. The switched large-scale time-delay system (1) is globally exponentially stable with stability margin $\lambda$ for $0<\tau<\lambda /\|E\|$, if the system (1) satisfies the following switching law:

$$
\inf _{t \geq t_{0}}\left[\frac{T^{-}(t)}{T^{+}(t)}\right] \geq \max _{1 \leq i \leq N}\left(\frac{T_{i}^{-}(t)}{T_{i}^{+}(t)}\right)
$$

where $\lambda=\min _{1 \leq i \leq N}\left(\lambda_{i}\right)$.

Proof. We can obtain the following equation:

$$
\begin{aligned}
x_{i}(t-\tau)= & x_{i}(t)-\int_{t-\tau}^{t} \dot{x}_{i}(s) d s \\
=x_{i}(t)-\int_{t-\tau}^{t}[ & \sum_{j=1}^{N} A_{l i j} x_{j}(s) \\
& \left.\quad+\sum_{j=1}^{N} B_{l_{i j}} x_{j}(s-\tau)\right] d s .
\end{aligned}
$$

Therefore, the switched large-scale time-delay system (1) can also be written as

$$
\begin{aligned}
\dot{x}_{i}(t)= & A_{l-i i} x_{i}(t)+\sum_{\substack{j \neq i \\
j=1}}^{N} A_{l i j} x_{j}(t) \\
& +\sum_{j=1}^{N} B_{l-i j}\left\{x_{l-j}(t)\right. \\
& -\int_{t-\tau}^{t}\left[\sum_{m=1}^{N} A_{l-j m} x_{m}(s)\right. \\
= & \bar{A}_{l-i i} x_{i}(t)+\sum_{\substack{j \neq i \\
j=1}}^{N} \bar{A}_{l i j} x_{j}(t) \\
& \left.-\sum_{j=1}^{N} B_{l-i j} \int_{t-\tau}^{t}\left[\sum_{m=1}^{N} A_{l-j m}(s-\tau) d s\right]\right\} \\
& \left.+\sum_{m=1}^{N} B_{l-j m} x_{m}(s-\tau) d s\right],
\end{aligned}
$$

where $\bar{A}_{l_{i i}}=A_{l_{-i i}}+B_{l i i i}$ and $\bar{A}_{l i j}=A_{l_{-i j}}+B_{l i j}$.
Hence, the solution of the system (10) can be expressed as

$x_{i}(t)$

$$
\begin{aligned}
& =e^{\bar{A}_{P_{d+1} j i}\left(t-t_{d}\right)} e^{\bar{A}_{P_{d}-i i}\left(t_{d}-t_{d-1}\right)} \cdots e^{\bar{A}_{p_{1}-i i}\left(t_{1}-t_{0}\right)} x_{i}\left(t_{0}\right) \\
& +\int_{t_{0}}^{t_{1}} e^{\bar{A}_{p_{d+1}}\left(t i t_{d}\right)} e^{\bar{A}_{P_{d} \cdot i i}\left(t_{d}-t_{d-1}\right)} \cdots e^{\bar{A}_{p_{1} \cdot i i}\left(t_{1}-s\right)} \\
& \times \sum_{\substack{j \neq i \\
j=1}}^{N} \bar{A}_{p_{1} i j} x_{j}(s) d s \\
& +\int_{t_{1}}^{t_{2}} e^{\bar{A}_{P_{d+1}}\left(t i t_{d}\right)} e^{\bar{A}_{P_{d}-i i}\left(t_{d}-t_{d-1}\right)} \cdots e^{\bar{A}_{P_{2}-i i}\left(t_{2}-s\right)}
\end{aligned}
$$$$
\times \sum_{\substack{j \neq i \\ j=1}}^{N} \bar{A}_{p_{2}-i j} x_{j}(s) d s
$$$$
+\int_{t_{d}}^{t} e^{\bar{A}_{p_{d+1}-i i}(t-s)} \sum_{\substack{j \neq i \\ j=1}}^{N} \bar{A}_{p_{d+1} i i} x_{j}(s) d s
$$$$
-\int_{t_{0}}^{t_{1}} e^{\bar{A}_{P_{d+1}}\left(t i-t_{d}\right)} \cdots e^{\bar{A}_{p_{1} i i}\left(t_{1}-s\right)}
$$$$
\times \sum_{j=1}^{N} B_{p_{1}-i j} \int_{s-\tau}^{s}\left[\sum_{m=1}^{N} A_{p_{1}-j m} x_{m}(\theta)\right.
$$$$
\left.+\sum_{m=1}^{N} B_{j m}^{p_{1}} x_{m}(\theta-\tau)\right] d \theta d s
$$$$
-\int_{t_{1}}^{t_{2}} e^{\bar{A}_{p_{d+1}} i i\left(t-t_{d}\right)} \cdots e^{\bar{A}_{p_{2} j i}\left(t_{2}-s\right)}
$$$$
\times \sum_{j=1}^{N} B_{p_{2}-i j} \int_{s-\tau}^{s}\left[\sum_{m=1}^{N} A_{p_{2}-j m} x_{m}(\theta)\right.
$$$$
\left.+\sum_{m=1}^{N} B_{p_{2}-j m} x_{m}(\theta-\tau)\right] d \theta d s
$$

$$
\begin{aligned}
- & \int_{t_{d}}^{t} e^{\bar{A}_{p_{d+1}} i i(t-s)} \sum_{j=1}^{N} B_{p_{d+1} i i} \\
\times \int_{s-\tau}^{s} & {\left[\sum_{m=1}^{N} A_{p_{d+1}-j m} x_{m}(\theta)\right.} \\
& \left.+\sum_{m=1}^{N} B_{p_{d+1}-j m} x_{m}(\theta-\tau)\right] d \theta d s,
\end{aligned}
$$


where $p_{d} \in\{1,2, \ldots, r\}$. Let

$$
v_{i}(t)=e^{\bar{A}_{p_{d+1} i i}\left(t-t_{d}\right)} e^{\bar{A}_{p_{d}-i i}\left(t_{d}-t_{d-1}\right)} \cdots e^{\bar{A}_{p_{1 i i}}\left(t_{1}-t_{0}\right)} v_{i}\left(t_{0}\right) .
$$

Performing the norm on both sides of (12) and in view of Lemma 1, we can obtain

$$
\begin{aligned}
\left\|v_{i}(t)\right\| \leq & e^{\left\|\bar{A}_{p_{d+1} i i}\left(t-t_{d}\right)\right\|} \\
& \times e^{\left\|\bar{A}_{p_{d} i i}\left(t_{d}-t_{d-1}\right)\right\|} \cdots e^{\left\|\bar{A}_{p_{1}-i i}\left(t_{1}-t_{0}\right)\right\|} v_{i}\left(t_{0}\right) \\
\leq & e^{\mu\left(A_{p_{i+1} i i}+B_{p_{i+1} i i}\right)\left(t-t_{i}\right)} \\
& \times e^{\mu\left(A_{p_{i} i i}+B_{p_{i} i i}\right)\left(t_{i}-t_{i-1}\right)} \cdots e^{\mu\left(A_{p_{1} i i}+B_{p_{1} i i}\right)\left(t_{1}-t_{0}\right)} \\
& \times\left\|v_{i}\left(t_{0}\right)\right\| \\
\leq & e^{\lambda_{i}^{+} T^{+}\left(t_{0}, t\right)-\lambda_{i}^{-} T\left(t_{0}, t\right)}\left\|v_{i}\left(t_{0}\right)\right\| .
\end{aligned}
$$

Furthermore, the switching law (6) of the ith subsystem can become as

$$
\lambda_{i}^{+} T_{i}^{+}(t)-\lambda_{i}^{-} T_{i}^{-}(t) \leq-\lambda_{i}^{*}\left(T_{i}^{+}(t)+T_{i}^{-}(t)\right)=-\lambda_{i}^{*}\left(t-t_{0}\right) .
$$

Hence, the following inequality can be obtained:

$$
\left\|v_{i}(t)\right\| \leq e^{-\lambda_{i}^{*}\left(t-t_{0}\right)}\left\|v_{i}\left(t_{0}\right)\right\| \leq e^{-\lambda_{i}\left(t-t_{0}\right)}\left\|v_{i}\left(t_{0}\right)\right\| .
$$

Using the properties of matrix norm, we can obtain the following inequality:

$$
\begin{aligned}
& r l \int_{s-\tau}^{s} B_{i}\left[A_{i} x(\theta)+B_{i} x(\theta-\tau)\right] d \theta \\
& \quad \leq \int_{s-\tau}^{s}\left[\left\|A_{i} B_{i}\right\|\|x(\theta)\|+\left\|B_{i}^{2}\right\|\|x(\theta-\tau)\|\right] d \theta \\
& \quad \leq \tau E_{i} \sup _{s-2 \tau \leq \theta \leq s}\|x(\theta)\|,
\end{aligned}
$$

where $E_{i}=\left\|A_{i} B_{i}\right\|+\left\|B_{i}^{2}\right\|, 1 \leq i \leq N$.
Hence, performing the norm on both sides of (11) and in view of Lemma 1, we can obtain

$$
\begin{aligned}
& \left\|x_{i}(t)\right\| \\
& \leq e^{-\lambda_{i}\left(t-t_{0}\right)}\left\|x_{i}\left(t_{0}\right)\right\| \\
& +\int_{t_{0}}^{t_{1}} e^{-\lambda_{i}(t-s)} \sum_{\substack{j \neq i \\
j=1}}^{N} a_{i j}^{*}\left\|x_{j}(s)\right\| d s \\
& +\int_{t_{1}}^{t_{2}} e^{-\lambda_{i}(t-s)} \sum_{\substack{j \neq i \\
j=1}}^{N} a_{i j}^{*}\left\|x_{j}(s)\right\| d s \\
& +\cdots+\int_{t_{d}}^{t} e^{-\lambda_{i}(t-s)} \sum_{\substack{j \neq i \\
j=1}}^{N} a_{i j}^{*}\left\|x_{j}(s)\right\| d s \\
& +\int_{t_{0}}^{t_{1}} e^{-\lambda_{i}(t-s)} \int_{s-\tau}^{s}\left[\sum _ { j = 1 } ^ { N } \sum _ { m = 1 } ^ { N } \left(\left\|B_{p_{1} i j} A_{p_{1-j m}}\right\|\right.\right. \\
& \times\left\|x_{m}(\theta)\right\|+\left\|B_{p_{1} i j} B_{p_{1-j} j m}\right\| \\
& \left.\left.\times\left\|x_{m}(\theta-\tau)\right\|\right)\right] d \theta d s \\
& +\int_{t_{1}}^{t_{2}} e^{-\lambda_{i}(t-s)} \int_{s-\tau}^{s}\left[\sum _ { j = 1 } ^ { N } \sum _ { m = 1 } ^ { N } \left(\left\|B_{p_{2}-i j} A_{p_{2}-j m}\right\|\right.\right. \\
& \begin{array}{l}
\times\left\|x_{m}(\theta)\right\|+\left\|B_{p_{2}-i j} B_{p_{2}-j m}\right\| \\
\left.\left.\times\left\|x_{m}(\theta-\tau)\right\|\right)\right] d \theta d s
\end{array}
\end{aligned}
$$

$$
\begin{aligned}
+\int_{t_{d}}^{t} e^{-\lambda_{i}(t-s)} \int_{s-\tau}^{s}\left[\sum_{j=1}^{N} \sum_{m=1}^{N}\right. & \left(\left\|B_{p_{d} i j} A_{p_{d}-j m}\right\|\right. \\
& \times\left\|x_{m}(\theta)\right\|+\left\|B_{p_{d} i j} A_{p_{d-j} j m}\right\| \\
& \left.\left.\times\left\|x_{m}(\theta-\tau)\right\|\right)\right] d \theta d s
\end{aligned}
$$$$
=e^{-\lambda_{i}\left(t-t_{0}\right)}\left\|x_{i}\left(t_{0}\right)\right\|+\int_{t_{0}}^{t} e^{-\lambda_{i}(t-s)} \sum_{\substack{j \neq i \\ j=1}}^{N} a_{i j}^{*}\left\|x_{j}(s)\right\| d s
$$$$
+\int_{t_{0}}^{t} e^{-\lambda_{i}(t-s)} \int_{s-\tau}^{s}\left[\sum _ { j = 1 } ^ { N } \sum _ { m = 1 } ^ { N } \left(\left\|B_{p_{d} i j} A_{p_{d-j} j m}\right\|\right.\right.
$$ 


$$
\begin{array}{r}
\times\left\|x_{m}(\theta)\right\|+\left\|B_{p_{d} i j} A_{p_{d}-j m}\right\| \\
\left.\left.\times\left\|x_{m}(\theta-\tau)\right\|\right)\right] d \theta d s \\
\leq e^{-\lambda_{i}\left(t-t_{0}\right)}\left\|x_{i}\left(t_{0}\right)\right\|+\int_{t_{0}}^{t} e^{-\lambda_{i}(t-s)} \sum_{\substack{j \neq i \\
j=1}}^{N} a_{i j}^{*}\left\|x_{j}(s)\right\| d s \\
+\tau \int_{t_{0}}^{t} e^{-\lambda(t-s)} \sum_{j=1}^{N} e_{i j}^{*} \sup _{s-2 \tau \leq \theta \leq s}\left\|x_{j}(\theta)\right\| d s .
\end{array}
$$

Let

$$
y_{i}(t)=e^{-\lambda_{i}\left(t-t_{0}\right)}\left\|y_{i}\left(t_{0}\right)\right\|+\int_{t_{0}}^{t} e^{-\lambda_{i}(t-s)} \sum_{\substack{j \neq i \\ j=1}}^{N} a_{i j}^{*}\left\|y_{j}(s)\right\| d s .
$$

Then, (18) is the solution of the following system:

$$
\dot{y}_{i}(t)=-\lambda_{i} y_{i}(t)+\sum_{\substack{j \neq i \\ j=1}}^{N} a_{i j}^{*} y_{j}(t)+\tau \sum_{j=1}^{N} e_{i j}^{*} \sup _{t-2 \tau \leq \theta \leq t} y_{j}(\theta) .
$$

Hence, the exponentially stability of $y_{i}(t)$ implies that of $x_{i}(t)$ by the comparison theorem. Furthermore, the system (19) also can be expressed as the system (7). It is clear that if the system (7) is stable and the switching law is as (8), then the switched large-scale time-delay system (1) is also globally exponentially stable with stability margin $\lambda$.

\section{An Illustrative Example}

Example 4. Consider a switched large-scale time-delay system composed of three individual switched systems.

Individual system $1(l=1)$ :

$$
\begin{aligned}
\dot{x}_{1}(t)= & {\left[\begin{array}{cc}
-8 & 2 \\
4 & -7
\end{array}\right] x_{1}(t)+\left[\begin{array}{ll}
-2 & -2 \\
-4 & -2
\end{array}\right] x_{1}(t-\tau) } \\
& +\left[\begin{array}{cc}
0.5 & 1 \\
0.5 & 0.2
\end{array}\right] x_{2}(t)+\left[\begin{array}{cc}
0.8 & 1 \\
0.1 & 0.5
\end{array}\right] x_{2}(t-\tau) \\
& +\left[\begin{array}{cc}
0 & -0.1 \\
-0.2 & 0.3
\end{array}\right] x_{3}(t)+\left[\begin{array}{cc}
-1 & -0.9 \\
-0.1 & -2
\end{array}\right] x_{3}(t-\tau), \\
\dot{x}_{2}(t)= & {\left[\begin{array}{cc}
0.2 & 0.7 \\
0.1 & 0.3
\end{array}\right] x_{1}(t)+\left[\begin{array}{cc}
1.1 & 0.2 \\
-0.5 & 0.7
\end{array}\right] x_{1}(t-\tau) } \\
& +\left[\begin{array}{cc}
-10 & 2 \\
0 & -12
\end{array}\right] x_{2}(t)+\left[\begin{array}{cc}
0.8 & 0.4 \\
-0.5 & -0.7
\end{array}\right] x_{2}(t-\tau) \\
& +\left[\begin{array}{cc}
1 & -0.3 \\
0.4 & 0
\end{array}\right] x_{3}(t)+\left[\begin{array}{cc}
-1 & 0.5 \\
0.7 & 0.9
\end{array}\right] x_{3}(t-\tau),
\end{aligned}
$$

$$
\begin{aligned}
\dot{x}_{3}(t)= & {\left[\begin{array}{cc}
0.1 & 0.7 \\
0.9 & -1
\end{array}\right] x_{1}(t)+\left[\begin{array}{cc}
2 & 0.3 \\
0.5 & 0.7
\end{array}\right] x_{1}(t-\tau) } \\
& +\left[\begin{array}{cc}
0 & 0.4 \\
1 & 0
\end{array}\right] x_{2}(t)+\left[\begin{array}{cc}
0.1 & -1 \\
-0.7 & 2
\end{array}\right] x_{2}(t-\tau) \\
& +\left[\begin{array}{cc}
-13 & 1 \\
0.5 & -12
\end{array}\right] x_{3}(t)+\left[\begin{array}{cc}
1.1 & 0.1 \\
0 & 0.5
\end{array}\right] x_{3}(t-\tau) .
\end{aligned}
$$

Individual system $2(l=2)$ :

$$
\begin{aligned}
\dot{x}_{1}(t)= & {\left[\begin{array}{cc}
1 & 0.5 \\
0.8 & 1.2
\end{array}\right] x_{1}(t)+\left[\begin{array}{cc}
-0.5 & 1 \\
0.7 & 0.9
\end{array}\right] x_{1}(t-\tau) } \\
& +\left[\begin{array}{cc}
-0.2 & 0 \\
0 & -0.3
\end{array}\right] x_{2}(t)+\left[\begin{array}{cc}
0.6 & 0.7 \\
0.1 & 1
\end{array}\right] x_{2}(t-\tau) \\
& +\left[\begin{array}{cc}
-1 & -0.2 \\
-0.5 & 1
\end{array}\right] x_{3}(t)+\left[\begin{array}{cc}
-0.1 & 0.2 \\
1 & 0.5
\end{array}\right] x_{3}(t-\tau), \\
\dot{x}_{2}(t)= & {\left[\begin{array}{cc}
0.7 & -0.1 \\
1 & -0.2
\end{array}\right] x_{1}(t)+\left[\begin{array}{cc}
-0.1 & 0.2 \\
0.2 & 1.1
\end{array}\right] x_{1}(t-\tau) } \\
& +\left[\begin{array}{cc}
-1 & 1 \\
0.5 & 0.8
\end{array}\right] x_{2}(t)+\left[\begin{array}{cc}
-2 & 1 \\
0.1 & 1
\end{array}\right] x_{2}(t-\tau) \\
& +\left[\begin{array}{cc}
0.2 & 0.1 \\
0.9 & 0.7
\end{array}\right] x_{3}(t)+\left[\begin{array}{cc}
0.1 & 1 \\
0.8 & 0.9
\end{array}\right] x_{3}(t-\tau), \\
\dot{x}_{3}(t)= & {\left[\begin{array}{cc}
-1.1 & 0.7 \\
0.1 & -0.4
\end{array}\right] x_{1}(t)+\left[\begin{array}{cc}
1.1 & 0.2 \\
0.7 & 0.9
\end{array}\right] x_{1}(t-\tau) } \\
& +\left[\begin{array}{cc}
-2 & 0.5 \\
-1 & -0.8
\end{array}\right] x_{2}(t)+\left[\begin{array}{cc}
0.7 & 0.8 \\
0.1 & -0.5
\end{array}\right] x_{2}(t-\tau) \\
& +\left[\begin{array}{cc}
-10 & 0 \\
1 & -8
\end{array}\right] x_{3}(t)+\left[\begin{array}{cc}
0.1 & 0.4 \\
0.3 & -0.5
\end{array}\right] x_{3}(t-\tau) .
\end{aligned}
$$

Individual system $3(l=3)$ :

$$
\begin{aligned}
\dot{x}_{1}(t)= & {\left[\begin{array}{cc}
-8 & 0 \\
0 & -9
\end{array}\right] x_{1}(t)+\left[\begin{array}{cc}
0.1 & 0.1 \\
-0.2 & 0.3
\end{array}\right] x_{1}(t-\tau) } \\
& +\left[\begin{array}{cc}
-0.5 & 0.1 \\
0.9 & -2
\end{array}\right] x_{2}(t)+\left[\begin{array}{cc}
-0.2 & 0.3 \\
0.2 & 0.5
\end{array}\right] x_{2}(t-\tau) \\
& +\left[\begin{array}{cc}
0.1 & 0.4 \\
0.5 & 0.6
\end{array}\right] x_{3}(t)+\left[\begin{array}{cc}
1 & -0.2 \\
0.1 & 0.3
\end{array}\right] x_{3}(t-\tau), \\
\dot{x}_{2}(t)= & {\left[\begin{array}{cc}
1 & 0.4 \\
-1 & -0.1
\end{array}\right] x_{1}(t)+\left[\begin{array}{cc}
0.1 & 0.8 \\
0.7 & -0.1
\end{array}\right] x_{1}(t-\tau) } \\
& +\left[\begin{array}{cc}
0.1 & 0.4 \\
0.6 & 1
\end{array}\right] x_{2}(t)+\left[\begin{array}{cc}
0.1 & -0.1 \\
0.2 & 0.4
\end{array}\right] x_{2}(t-\tau) \\
& +\left[\begin{array}{cc}
1 & 0.7 \\
0.5 & -1
\end{array}\right] x_{3}(t)+\left[\begin{array}{cc}
0.1 & -0.2 \\
1 & 1
\end{array}\right] x_{3}(t-\tau),
\end{aligned}
$$




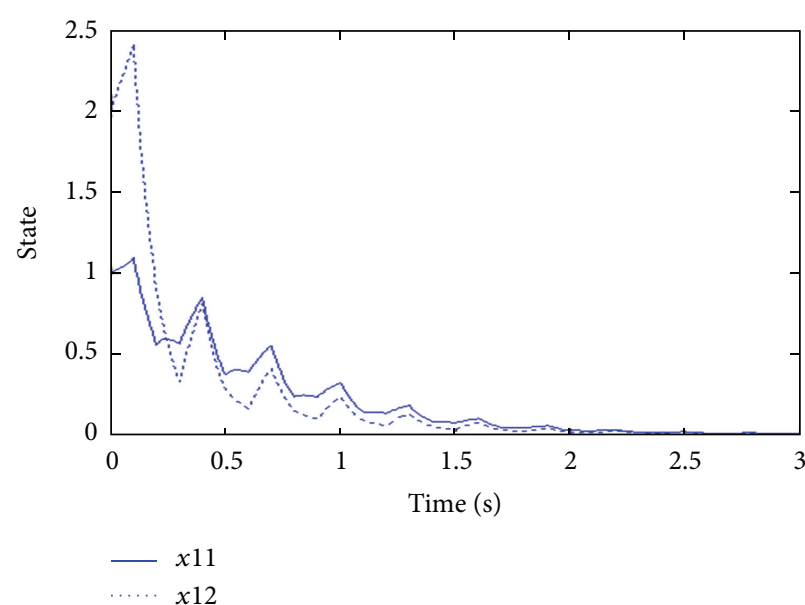

(a) State response for $x_{1}$

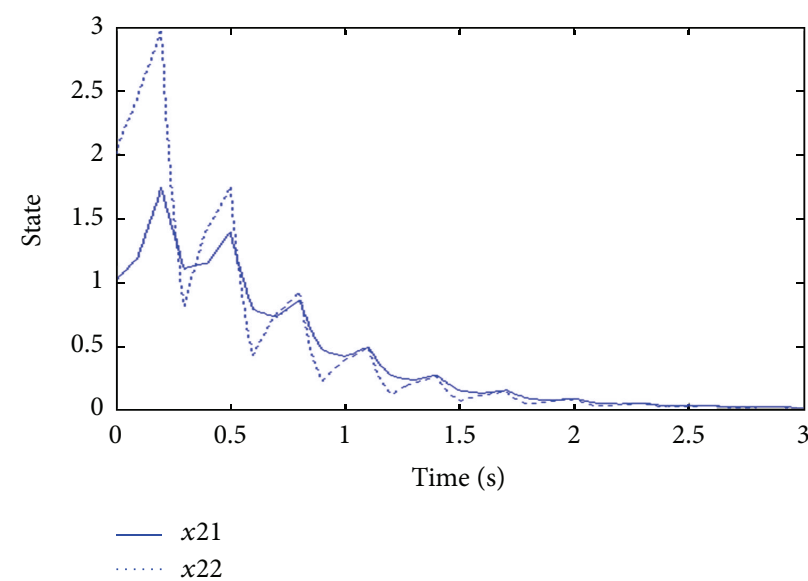

(b) State response for $x_{2}$

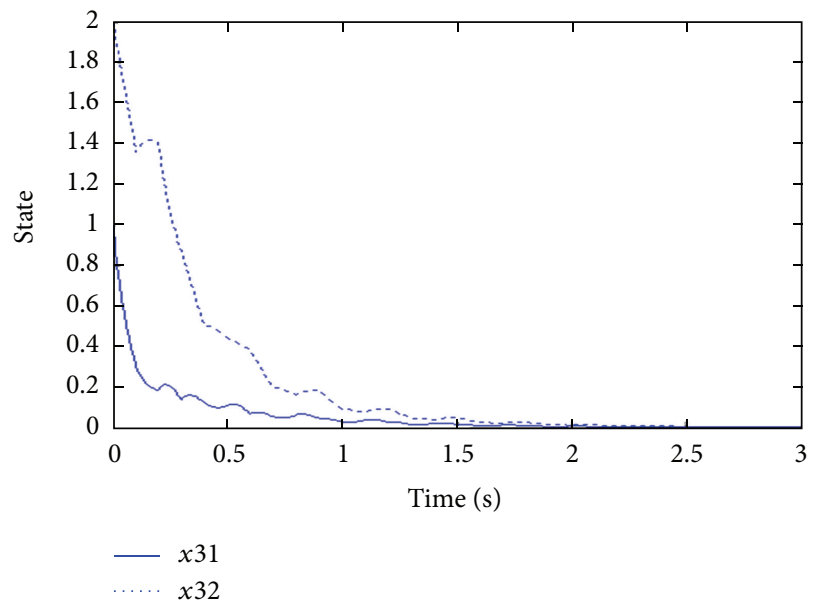

(c) State response for $x_{3}$

FIGURE 1: Trajectory of the time-delay switched system.

$$
\begin{aligned}
\dot{x}_{3}(t)= & {\left[\begin{array}{cc}
-0.1 & -0.1 \\
0.5 & 1
\end{array}\right] x_{1}(t)+\left[\begin{array}{cc}
0.1 & 0.5 \\
-0.1 & 0.8
\end{array}\right] x_{1}(t-\tau) } \\
& +\left[\begin{array}{cc}
0.2 & 0.3 \\
0.1 & 0.2
\end{array}\right] x_{2}(t)+\left[\begin{array}{cc}
0.1 & 0.1 \\
-1 & -0.5
\end{array}\right] x_{2}(t-\tau) \\
& +\left[\begin{array}{cc}
0.4 & 0.1 \\
-1 & -2
\end{array}\right] x_{3}(t)+\left[\begin{array}{cc}
-1 & -2 \\
1 & 0.5
\end{array}\right] x_{3}(t-\tau)
\end{aligned}
$$

According to the normal test of stability for the largescale system, the system 1 is stable; the systems 2 and 3 are both unstable. We can easily calculate $\lambda_{1}^{-}=7.8969, \lambda_{1}^{+}=$ $3, \lambda_{2}^{-}=8.9588, \lambda_{2}^{+}=2.1295$, and $\lambda_{3}^{-}=8.0989$ and $\lambda_{3}^{+}=$ 0.0012. Hence, the stable matrices $C=\left[\begin{array}{ccc}-5 & 2.5548 & 2.1329 \\ 1.6652 & -6 & 2.5398 \\ 2.6190 & 2.1041 & -6\end{array}\right]$ and $E=\left[\begin{array}{llll}48.4967 & 31.1791 & 36.7386 \\ 16.5155 & 15.0482 & 16.3642 \\ 21.6043 & 32.7876 & 18.1580\end{array}\right]$ can be obtained by choosing $\lambda_{1}=5, \lambda_{2}=6, \lambda_{3}=6, \lambda_{1}^{*}=5.5, \lambda_{2}^{*}=6.5$, and $\lambda_{3}^{*}=6.5$. Hence, $c=\mu(C)=-0.7516$ and $\|E\|=84.3058$. Furthermore, the switching law of the $i$ th subsystem, $i=$
$1,2,3$, can be calculated as $T_{1}^{-} / T_{1}^{+} \geq 3.5462, T_{2}^{-} / T_{2}^{+} \geq 3.5096$, and $T_{3}^{-} / T_{3}^{+} \geq 4.0660$. Therefore, the total activation time ratio for the switching law is $T^{-}(t) / T^{+}(t) \geq 4.0660$.

Finally, the switched large-scale time-delay system (22) is globally exponentially stable under the switching law with stability margin $\lambda=5$ and $0<\tau<0.013$. To satisfy the switching law, we choose the total activation time ratio to be $4.5: 1$. The activation time of individual system 1 is 4.5 seconds, and the total activation time for both systems 2 and 3 is 1 second. The trajectory of the large-scale time-delay-switched system is shown in Figure 1 with initial state $\left[\begin{array}{llllll}1 & 2 & 1 & 2 & 1 & 2\end{array}\right]^{T}$ and time delay being $0.01 \mathrm{sec}-$ ond.

\section{Conclusions}

From an analysis based on the time-switched method and the comparison theorem, a sufficient condition of the switched laws is presented. The total activation time ratio under the 
switching laws needs to be greater or equal to a specified constant to ensure that the switched large-scale time-delay system is delay-dependent exponentially stable with stability $\operatorname{margin} \lambda$.

\section{Acknowledgment}

This work is supported by the National Science Council, Taiwan, under Grant nos. NSC101-2221-E-218-027 and NSC 100-2632-E-218-001-MY3.

\section{References}

[1] D. Liberzon and A. S. Morse, "Basic problems in stability and design of switched systems," IEEE Control Systems Magazine, vol. 19, no. 5, pp. 59-70, 1999.

[2] Z. Ji, L. Wang, G. Xie, and F. Hao, "Linear matrix inequality approach to quadratic stabilisation of switched systems," IEE Proceedings: Control Theory and Applications, vol. 151, no. 3, pp. 289-294, 2004.

[3] J. S. Chiou and C. M. Cheng, "Stabilization analysis of the switched discrete-time systems using Lyapunov stability theorem and genetic algorithm," Chaos, Solitons and Fractals, vol. 42, no. 2, pp. 751-759, 2009.

[4] J. S. Chiou and C. J. Wang, "Stability analysis and timeswitching rule design for the switched continuous-time and discrete-time systems," International Journal of Nonlinear Sciences and Numerical Simulation, vol. 11, pp. 115-118, 2010.

[5] J. P. Hespanha and A. S. Morse, "Stability of switched systems with average dwell-time," in Proceedings of the 38th IEEE Conference on Decision and Control (CDC), pp. 2655-2660, Phoenix, Ariz, USA, December 1999.

[6] G. Zhai, B. Hu, K. Yasuda, and A. N. Michel, "Stability analysis of switched systems with stable and unstable subsystems: an average dwell time approach," International Journal of Systems Science, vol. 32, no. 8, pp. 1055-1061, 2001.

[7] S. H. Lee, T. H. Kim, and J. T. Lim, "New stability analysis of switched systems," Automatica, vol. 36, no. 6, pp. 917-922, 2000.

[8] C. D. Persis, R. D. Santis, and A. S. Morse, "Switched nonlinear systems with state-dependent dwell-time," Systems and Control Letters, vol. 50, no. 4, pp. 291-302, 2003.

[9] J. S. Chiou, C. J. Wang, and C. M. Cheng, "On delay-dependent stabilization analysis for the switched time-delay systems with the state-driven switching strategy," Journal of the Franklin Institute, vol. 348, no. 2, pp. 261-276, 2011.

[10] C. J. Wang, J. S. Chiou, and C. M. Cheng, "On delay-independent stabilization analysis for a class of switched time-delay systems with the state-driven switching strategy," Journal of the Franklin Institute, vol. 348, no. 9, pp. 2292-2307, 2011.

[11] X. Xiao and Z. Mao, "Decentralized guaranteed cost stabilization of time-delay large-scale systems based on reduced-order observers," Journal of the Franklin Institute, vol. 348, no. 9, pp. 2689-2700, 2011.

[12] J. S. Chiou, "Stability analysis for a class of switched large-scale time-delay systems via time-switched method," IEE Proceedings: Control Theory and Applications, vol. 153, no. 6, pp. 684-688, 2006.

[13] P. Lancaster, Theory of Matrices, Academic Press, New York, NY, USA, 1969.
[14] V. B. Kolmanovskii and V. R. Nosov, Stability of Functional Differential Equations, vol. 180 of Mathematics in Science and Engineering, Academic Press, London, UK, 1986. 


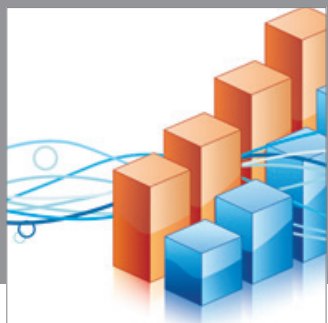

Advances in

Operations Research

mansans

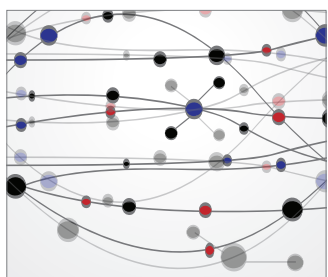

The Scientific World Journal
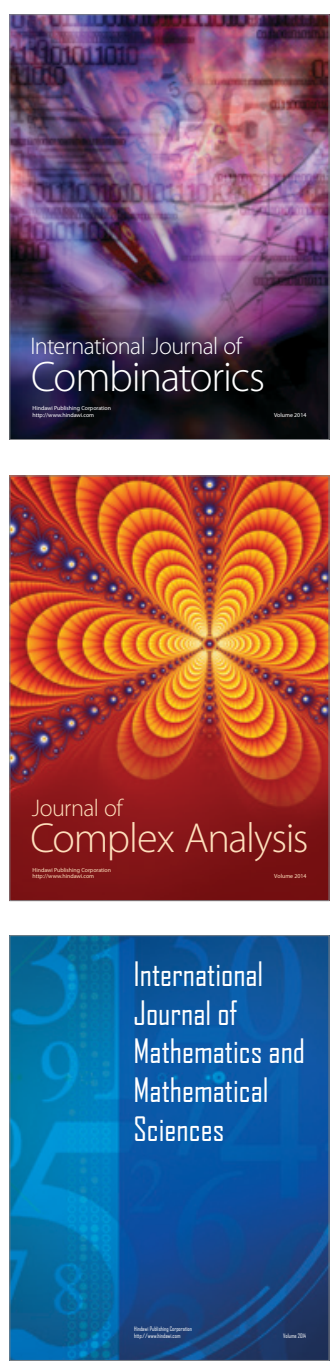
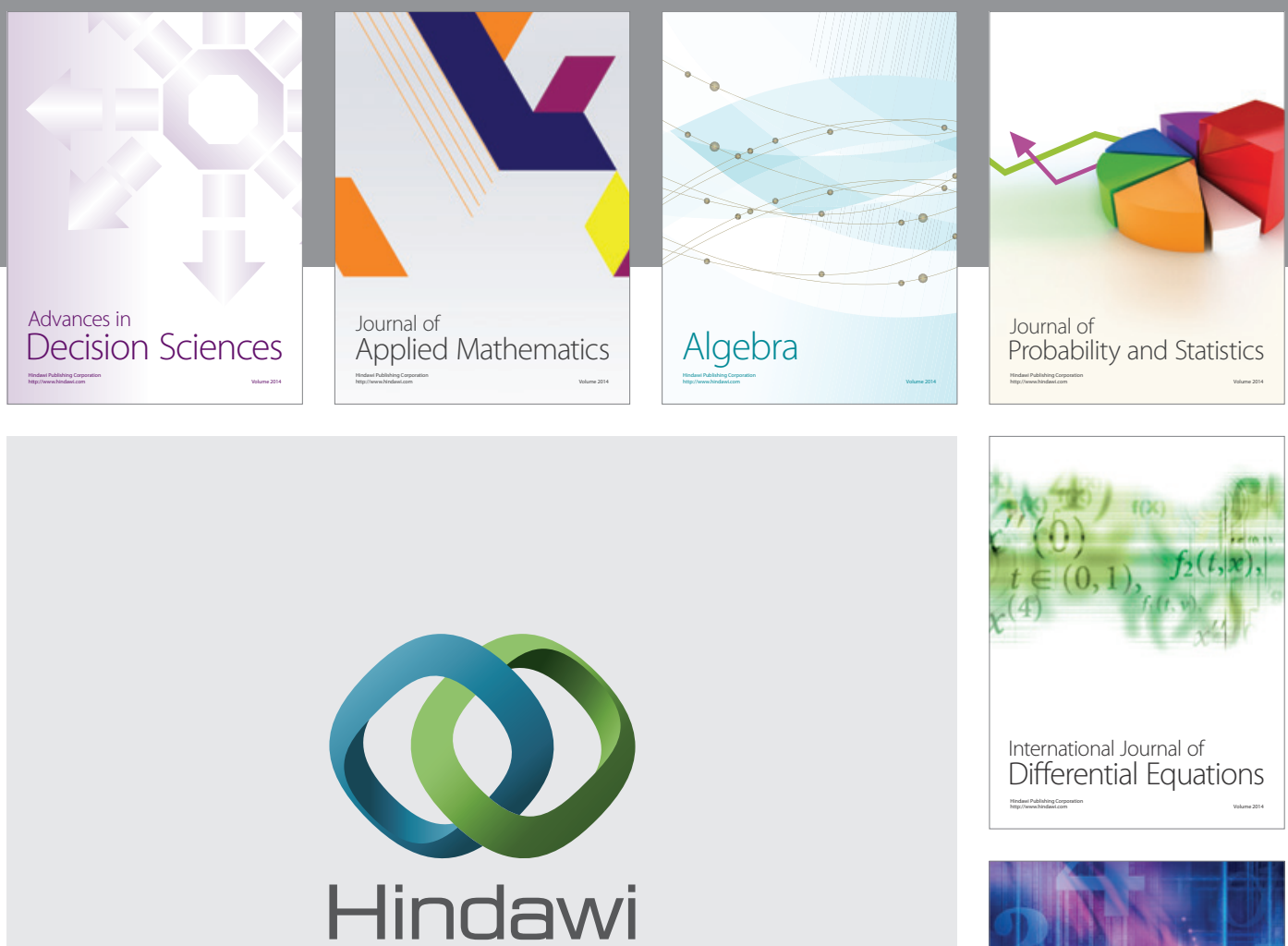

Submit your manuscripts at http://www.hindawi.com
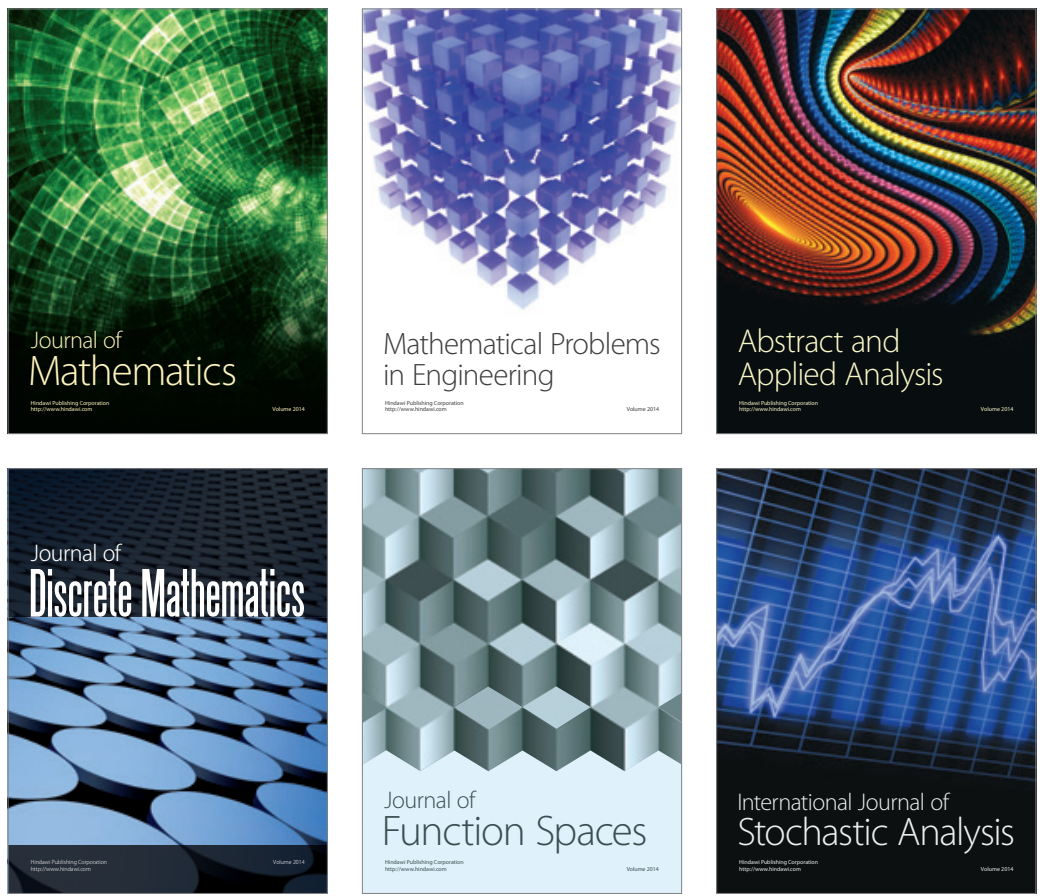

Journal of

Function Spaces

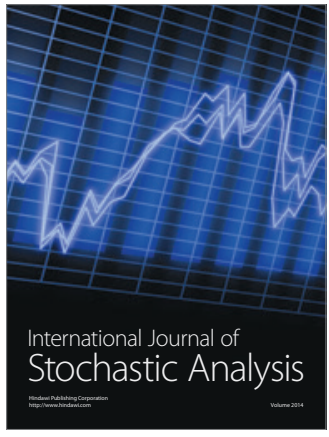

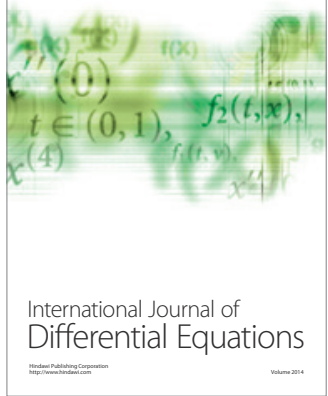
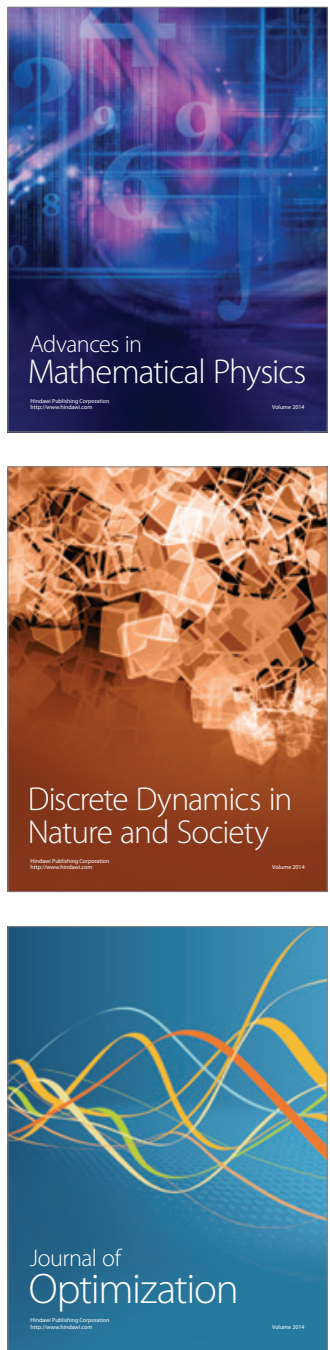